\title{
Existence results and Hyers-Ulam stability to a class of nonlinear arbitrary order differential equations
}

\author{
Poom Kumam ${ }^{a, b, c, *}$, Amjad Alid, Kamal Shah ${ }^{d}$, Rahmat Ali Khan ${ }^{d}$ \\ ${ }^{a}$ KMUTT-Fixed Point Research Laboratory, Department of Mathematics, Room SCL 802 Fixed Point Laboratory, Science Laboratory \\ Building, Faculty of Science, King Mongkut's University of Technology Thonburi (KMUTT), 126 Pracha Uthit Road, Bang Mod, Thung \\ Khru, Bangkok 10140, Thailand. \\ ${ }^{b}$ KMUTT-Fixed Point Theory and Applications Research Group, Theoretical and Computational Science (TaCS) Center, Science \\ Laboratory Building, Faculty of Science, King Mongkut's University of Technology Thonburi (KMUTT), 126 Pracha Uthit Road, Bang \\ Mod, Thung Khru, Bangkok 10140, Thailand. \\ ${ }^{c}$ Department of Medical Research, China Medical University Hospital, China Medical University, Taichung 40402, Taiwan. \\ ${ }^{d}$ Department of Mathematics, University of Malakand, Chakadara Dir(L), Khyber Pakhtunkhwa, Pakistan.
}

Communicated by B. Samet

\begin{abstract}
This paper is concerned with developing some conditions that reveal existing and stability analysis for solutions to a class of differential equations with fractional order. The required conditions are obtained by applying the technique of degree theory of topological type. The concerned problem is converted to the integral equation and then to operator equation, where the operator is defined by $T: C[0,1] \rightarrow C[0,1]$. It should be noted that the assumptions on nonlinear function $f(t, u(t))$ does not usually ascertain that the operator T being compact. Moreover, in this paper we also establish some conditions under which the solution of the considered class is Hyers-Ulam stable and also satisfies the conditions of Hyers-Ulam-Rassias and generalized Hyers-Ulam stability. Proper example is provided for the illustration of main results. (c)2017 All rights reserved.
\end{abstract}

Keywords: Arbitrary order differential equations, topological degree theory, condensing mapping, existence results, stability analysis.

2010 MSC: 34A08, 35R11, 26A33.

\section{Introduction and preliminaries}

Arbitrary order differential equations have attracted the attentions very well in last few decades. Because, non-integer order differential equations have many applications in various branches of science and engineering such as signal processing, viscoelasticity, biology, physics, chemistry, control theory and stability of networking and modeling of biological phenomenons, etc., for detail see $[6,13,14,16,18]$. The qualitative theory devoted to existence of solutions to non-integer order differential equations involving

\footnotetext{
*Corresponding author

Email addresses: poom.kum@kmutt.ac.th (Poom Kumam), amjadalimna@yahoo.com (Amjad Ali), kamalshah408@gmail.com (Kamal Shah), rahmat_alipk@yahoo.com (Rahmat Ali Khan)
}

doi:10.22436/jnsa.010.06.13 
boundary conditions has been an active area of research for the last few decades. By using various tools and method of functional analysis and fixed point theory, the concerned theory has been explored very well, for detail see $[1,2,22,23]$. However, in the mentioned papers, the concerned conditions for existence of solutions required compactness of the operator which restrict the area to some limited classes of fractional differential equations. To relax the conditions, one needs weaker condition for compactness of the operator T. One such powerful tool is a topological degree theory which was applied by Mawhin [15] and established essential conditions for the solutions of a classical differential and integral equation. Isaia [10] used topological degree method and developed some useful conditions for the existence theory of solutions to some nonlinear integral equations. In 2013, Wang et al. [29], established an existence theory for the solutions to a nonlocal Cauchy problems given below by applying topological degree method

$$
\left\{\begin{array}{l}
{ }^{c} D^{q} u(t)-f(t, u(t))=0, \quad t \in[0, T], \\
u(0)-u_{0}=g(u),
\end{array}\right.
$$

where ${ }^{c} \mathrm{D}^{\mathrm{q}}$ represents the Caputo fractional derivative of order $\mathrm{q} \in(0,1], \mathrm{u}_{0} \in \mathbb{R}$ and $\mathrm{f}: \mathrm{I} \times \mathbb{R} \rightarrow \mathbb{R}$ is continuous. In 2015, Khan and Shah [12], extended the topological degree method for the following multi-point boundary value problems given as

$$
\left\{\begin{array}{l}
{ }^{c} D^{q} u(t)-f(t, u(t))=0, \quad q \in(1,2], \quad t \in[0,1], \\
u(0)-g(u)=0, u(1)-\sum_{i=1}^{m-2} \lambda_{i} u\left(\eta_{i}\right)-h(u)=0, \quad \lambda_{i}, \eta_{i} \in(0,1),
\end{array}\right.
$$

where $g, h:([0,1], \mathbb{R})$ and $f \in([0,1] \times \mathbb{R}, \mathbb{R})$.

Another aspect of the qualitative theory which is very important from optimization and numerical point of view is devoted to stability analysis of the solutions to differential equations of fractional order. In this regard, Hyers-Ulam type stability for the solutions of differential equations of non-integer order has been introduced in many articles. In 1940, Ulam [26] raised a question that "Under what conditions does there exist an additive mapping near an approximately additive mapping?" This question gave birth to the initiation of the area to investigate stability for functional, integral and differential equations. In this regard Hyers [8] was the first mathematician who answered the Ulam's question for the additive mapping in complete normed spaces. Latter on, from 1982 to 1998, Rassias [19] developed the conditions under which linear and nonlinear mappings are Hyers-Ulam stable. Jung [11], established Hyers-Ulam stability for nonlinear mapping on a restricted domains. The first author who investigated the HyersUlam stability for linear differential equation in 1997 was Obloza [17]. Now enough literature can be found about the aforesaid area. For classical order differential equations the area has been well studied and plenty of paper can be found on it, few of them we refer in [20, 21, 28, 30]. However for non-integer order differential equations, the area has not yet properly explored and required further exploration. Recently very few articles on Hyers-Ulam stability for the solutions of differential equations of arbitrary order have been published which we refer in $[3,4,7,24,25,27,31]$. Motivated by the aforesaid work, this article is concerned to investigate existence and stability of solutions for fractional differential equations of arbitrary order which have boundary conditions involving fractional order derivative in the following form

$$
\left\{\begin{array}{l}
{ }^{c} D^{q} u(t)=f(t, u(t)), \quad q \in(1,2], \quad t \in J=[0,1], \\
u(0)=\delta^{c} D^{p} u(\eta)+g(u), \\
u(1)=\lambda{ }^{c} D^{p} u(\xi)+h(u), \quad \delta, \lambda, \eta, \xi \in \mathbb{R} \text { such that } \delta>\lambda, \quad \eta>\xi,
\end{array}\right.
$$

where $p \in(0,1)$ and $f: J \times \mathbb{R} \rightarrow \mathbb{R}$ is nonlinear continuous function. Further, the nonlocal function defined by $g, h:[0,1] \rightarrow \mathbb{R}$ are also continuous.

Motivated by the aforesaid study, we investigate the concerned stability for the solution of problem (1.1) under consideration. We study different kinds of stability to the proposed problem like Hyers-Ulam 
stability, generalized Hyers-Ulam Rassias stability and Rassias stability. Here we remark that the aforesaid stability has not investigated for muti-point boundary value problems involving fractional derivative in their boundary conditions. The whole results are demonstrated by providing a suitable example in the last section of this article.

\section{2. auxiliary results and definitions}

This section, deal with few basic definitions, lemmas and notations which can be found in $[5,10,13$, 16 , 18]. All the continuous functions from $J \rightarrow \mathbb{R}$ form a Banach space endowed with a topological norm $\|\mathfrak{u}\|_{\mathfrak{c}}=\sup \{|\mathfrak{u}(\mathrm{t})|: t \in[0,1]\}$. This space is denoted by $\mathcal{B}=\mathrm{C}(\mathrm{J}, \mathbb{R})$.

Definition 2.1. If $\phi \in \mathrm{L}^{1}([0, T], \mathbb{R})$, then the fractional order integral of order $q \in \mathbb{R}_{+}$is defined by

$$
I^{\mathrm{q}} \phi(\mathrm{t})=\int_{0}^{\mathrm{t}} \frac{(\mathrm{t}-\mathrm{s})^{\mathrm{q}-1}}{\Gamma(\mathrm{q})} \phi(\mathrm{s}) \mathrm{ds}
$$

provided that the integral on the right side is point wise defined on $(0, \infty)$.

Definition 2.2. The fractional derivative in the Caputo sense of non-integer order for a function $\phi(t)$ on $[0, T]$ is defined by

$$
{ }^{c} D^{q} \phi(t)=\int_{0}^{t} \frac{(t-s)^{n-q-1}}{\Gamma(n-q)} \phi^{(n)}(s) d s,
$$

where $\mathrm{n}-[\mathrm{q}]=1$, where $[\mathrm{q}]$ is the greatest integer function not greater than $\mathrm{q}$.

Theorem 2.3. The unique solution of the arbitrary order differential equation given below

$$
{ }^{c} D^{q} h(t)=0 \text {, where } q \in(n-1, n],
$$

is given by

$$
h(t)=d_{1}+d_{2} t+d_{3} t^{2}+\cdots+d_{n} t^{n-1} \text {, where } d_{j} \in \mathbb{R}, j=1,2,3, \cdots, n .
$$

Theorem 2.4. The result given below

$$
I^{q c} D^{q} h(t)=h(t)+d_{1}+d_{2} t+d_{3} t^{2}+\cdots+d_{n} t^{n-1},
$$

holds for $\mathrm{d}_{\mathrm{j}} \in \mathbb{R}$, where $\mathrm{j}=1,2,3, \ldots, \mathrm{n}$.

We recall the following definitions and results needed in this study from Deimling [5]. Taking a family $\mathrm{U} \subset \mathrm{P}(\mathcal{B})$ of all bounded sets, then the measure of non-compactness of Kuratowski type is recalled below.

Definition 2.5. The mapping $\alpha: U \rightarrow \mathbb{R}^{+}$is introduced by

$$
\alpha(D)=\inf \{r>0: D \text { insert a finite cover by sets of diameter } \leqslant r, D \in U\} .
$$

We say that if $S \subset \mathcal{B}$ and $\mathfrak{F}: S \rightarrow \mathcal{B}$ is a continuous and bounded function, then $\mathfrak{F}$ is $\alpha$-Lipschitz if we have a constant $\kappa \geqslant 0$ for all bounded sets such that

$$
\alpha(\mathfrak{F}(\mathbb{S})) \leqslant \kappa \alpha(\mathbb{S}) .
$$

Further, if $\kappa<1$, then the function $\mathfrak{F}$ is called strict $\alpha$-condensing mapping and we have $\alpha(\mathfrak{F}(\mathbb{S}))<\alpha(\mathbb{S})$.

For the aforesaid contraction, we recall some properties without proof.

Theorem 2.6. If $\mathfrak{F}, \mathfrak{G}: S \rightarrow \mathcal{B}$ are $\alpha$-Lipschitz with constants $\kappa, \kappa^{\prime}$. The sum of the two operators $\mathfrak{F}+\mathfrak{G}: S \rightarrow \mathcal{B}$ is also $\alpha$-Lipschitz with constant $\mathrm{K}+\mathrm{K}^{\prime}$. 
Theorem 2.7. The mapping $\mathfrak{G}: S \rightarrow \mathcal{B}$ will be $\alpha$-Lipschitz with constant 0 , if it is compact.

Theorem 2.8. Let $\mathfrak{F}: S \rightarrow \mathcal{B}$ is Lipschitz with constant $\kappa$, then it will be $\alpha$-Lipschitz with constant $\kappa$.

Let a family of the admissible triplet defined by

$$
\mathfrak{I}=\{(\mathrm{I}-\mathfrak{T}, \mathrm{S}, \mathrm{u}): \mathfrak{S} \subset \mathcal{B} \text { be open and bounded, } \mathfrak{T} \text { is } \alpha \text {-condensing, } \mathfrak{u} \in \mathcal{B} \backslash(\mathrm{I}-\mathfrak{T})(\partial \mathrm{S})\} \text {. }
$$

Then there exists one degree function $\operatorname{deg}: \mathfrak{I} \rightarrow \mathcal{B}$ for which we have the following important result given by Isaia [10].

Theorem 2.9. Let $\mathfrak{T}: \mathcal{B} \rightarrow \mathcal{B}$ be $\alpha$-contraction and

$$
\mathcal{U}=\{\mathfrak{u} \in \mathcal{B}: \text { we have } 0 \leqslant \lambda \leqslant 1 \text {, which satisfies the eigenvalues problem } \mathrm{u}(\mathrm{t})=\lambda \mathfrak{T} u(t)\} \text {. }
$$

Consider a bounded set $\mathbb{U} \subset \mathcal{B}$ such that there exists $\epsilon>0$ with $\mathbb{U} \subset S_{\epsilon}(0)$, then

$$
\operatorname{deg}\left(\mathrm{I}-\lambda \mathfrak{F}, \mathrm{S}_{\epsilon}(0), 0\right)=1, \quad \forall \lambda \in[0,1] .
$$

Therefore $\mathfrak{T}$ keeps at least one fixed point and the set of the fixed points of $\mathfrak{T}$ lies in $S_{\epsilon}(0)$.

Definition 2.10. The class of boundary value problem (1.1) is Hyers-Ulam stable if there exists a real constant $C_{f}>0$, such that for $\varepsilon>0$, and for every solution $u \in \mathcal{B}$ of the inequality

$$
\left|{ }^{c} D^{q} u(t)-f(t, u(t))\right| \leqslant \varepsilon, \quad t \in J,
$$

there exists a solution $v \in \mathcal{B}$ of BVP (1.1) with

$$
|u(t)-v(t)| \leqslant C_{f} \varepsilon, \quad t \in J .
$$

Definition 2.11. The class of boundary value problem (1.1) is generalized Hyers-Ulam stable if one has a function $\Phi_{f} \in\left(\mathbb{R}^{+}, \mathbb{R}^{+}\right)$, with $\Phi_{f}(0)=0$ such that for every solution $u \in \mathcal{B}$ of equation (2.1), there exists a solution $v \in \mathcal{B}$ of BVP (1.1) which satisfies the following inequality:

$$
|u(t)-v(t)| \leqslant \Phi_{f}(\varepsilon), \quad t \in J .
$$

Definition 2.12. The class of boundary value problem (1.1) is Hyers-Ulam-Rassias stable with respect to $\psi: J \rightarrow \mathbb{R}^{+}$, if there exists a real constant $C_{f}>0$, such that for every $\varepsilon>0$, and for every solution $u \in \mathcal{B}$ of the inequality

$$
\left|{ }^{c} D^{q} u(t)-f(t, u(t))\right| \leqslant \varepsilon \psi(t), \quad t \in J,
$$

there exists a solution $v \in \mathcal{B}$ of BVP (1.1) with

$$
|u(t)-v(t)| \leqslant C_{f} \varepsilon \psi(t), \quad t \in J .
$$

Definition 2.13. The class of boundary value problem (1.1) is generalized Hyers-Ulam-Rassias stable with respect to $\psi: J \rightarrow \mathbb{R}^{+}$, if there exists a real constant $C_{f, \psi}>0$ such that for every $\varepsilon>0$ and for every $u \in \mathcal{B}$ of the inequality

$$
\left|{ }^{c} D^{q} u(t)-f(t, u(t))\right| \leqslant \psi(t), \quad t \in J,
$$

there exists a solution $v \in \mathcal{B}$ of BVP (1.1) with

$$
|u(t)-v(t)| \leqslant C_{f, \phi} \psi(t), \quad t \in J .
$$

\section{Existence and uniqueness results to BVP (1.1)}

Theorem 3.1. Assume that $\mathrm{y} \in([0,1], \mathbb{R}), \delta>\lambda, \eta>\xi$ are positive real numbers, then the general solution of the boundary value problem

$$
\left\{\begin{array}{l}
{ }^{c} D^{q} u(t)-y(t)=0, \quad q \in(0,1], \quad t \in J=[0,1], \\
u(0)=\delta^{c} D^{p} u(\eta)+g(u), \\
u(1)=\lambda{ }^{c} D^{p} u(\xi)+h(u), \quad \delta>\lambda, \quad \eta>\xi, 0<p<1,
\end{array}\right.
$$


is given by

$$
u(t)=l(t+m) h(u)+(1-l(t+m)) g(u)+\int_{0}^{1} \mathcal{H}(t, s) y(s) d s,
$$

such that the Green's function $\mathcal{H}(t, s)$ is given by

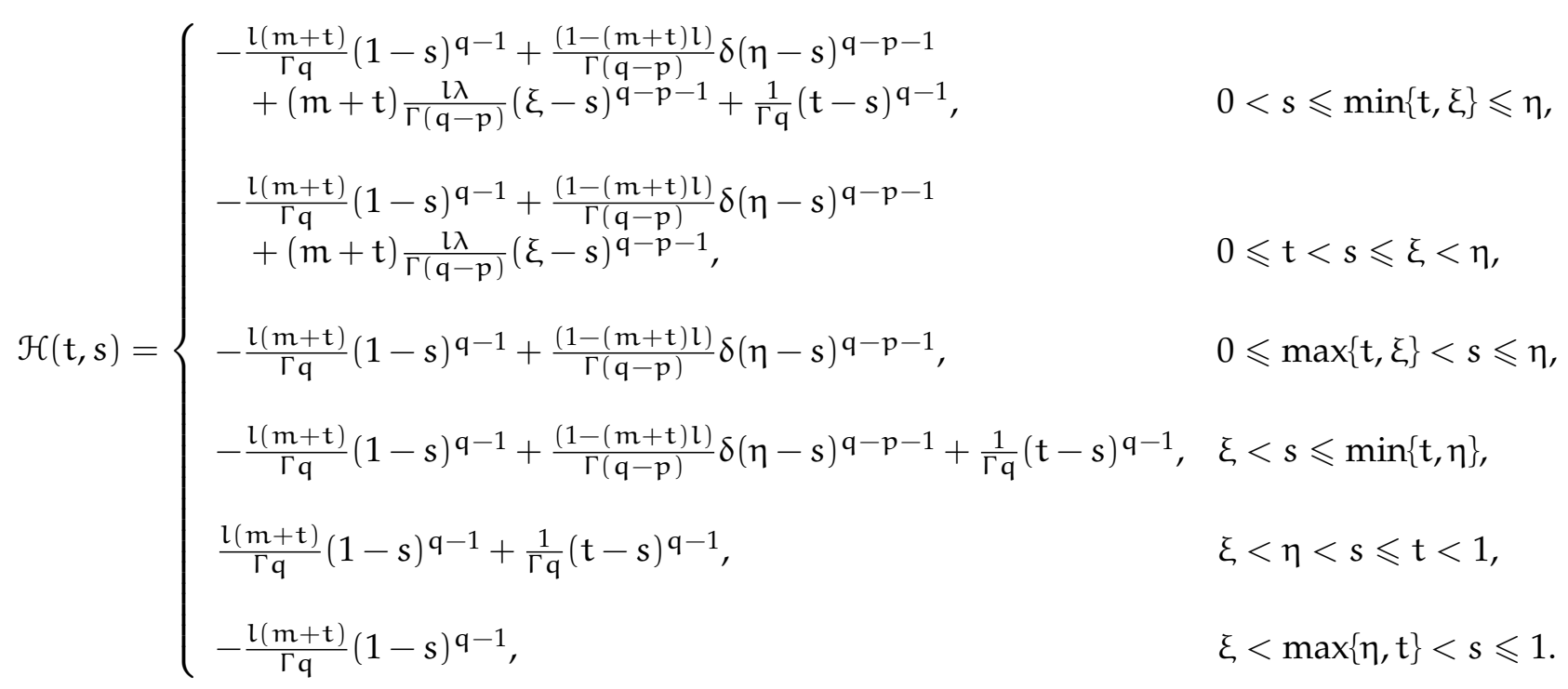

Proof. Consider ${ }^{c} D^{q} u(t)=y(t)$. By the application of Theorem 2.4, we obtain

$$
u(t)=d_{1}+d_{2} t+I^{q} y(t) \text {, and }{ }^{c} D^{q} u(t)=\frac{d_{2} t^{1-p}}{\Gamma(2-p)}+I^{q-p} y(t) .
$$

Using

$$
u(0)=\delta^{c} D^{p} u(\eta)+g(u)
$$

we get

$$
d_{1}=\frac{\delta \eta^{1-p}}{\Gamma(2-p)} d_{2}+\delta I^{q-p} y(\eta)+g(u)
$$

and

$$
u(1)=\lambda^{c} D^{p} u(\xi)+h(u)
$$

we have

$$
d_{1}+d_{2}+I^{q} y(1)=\frac{\lambda \xi^{1-p}}{\Gamma(2-p)} d_{2}+\lambda I^{q-p} y(\xi)+h(u) .
$$

Solving for $d_{1}$ and $d_{2}$, we obtain

$$
\begin{aligned}
& d_{2}=l\left(-I^{q} y(1)-\delta I^{q-p} y(\eta)+\lambda I^{q-p} y(\xi)+h(u)-g(u)\right), \\
& d_{1}=m l I^{q} y(1)+\delta(1-m l) I^{q-p} y(\eta)+\lambda m l I^{q-p} y(\xi)+m l h(u)+(1-m l) g(u),
\end{aligned}
$$

where

$$
0<l=\frac{1}{\left[1+\frac{\delta \eta^{1-p}-\lambda \xi^{1-p}}{\Gamma(2-p)}\right]}<1, \quad \text { and } \quad 0<m=\frac{\delta \eta^{1-p}}{\Gamma(2-p)}<1
$$

Therefore

$$
u(t)=l(t+m) h(u)+(1-l(t+m)) g(u)-(t+m) \frac{l}{\Gamma(q)} \int_{0}^{1}(1-s)^{q-1} y(s) d s
$$




$$
\begin{aligned}
& +\frac{(1-(m+t) l) \delta}{\Gamma(q-p)} \int_{0}^{\eta}(\eta-s)^{q-p-1} y(s) d s+\frac{(m+t) l \lambda}{\Gamma(q-p)} \int_{0}^{\xi}(\xi-s)^{q-p-1} y(s) d s \\
& +\int_{0}^{t} \frac{(t-s)^{q-1}}{\Gamma(q)} y(s) d s \\
& =l(m+t) h(u)+(1-(m+t) l) g(u)+\int_{0}^{1} \mathcal{H}(t, s) y(s) d s,
\end{aligned}
$$

where $\mathcal{H}$ is the Green's function defined in (3.1).

Hence in view of Theorem 3.1, the solution of our considered class (1.1) can be received as

$$
u(t)=l(t+m) h(u)+(1-l(t+m)) g(u)+\int_{0}^{1} \mathcal{H}(t, s) f(s, u(s)) d s,
$$

where, the function $t: \longrightarrow \int_{0}^{1}|\mathcal{H}(t, s)| d s$ is also continuous on J. Denoting $\mathcal{H}^{*}=\sup _{t \in J} \int_{0}^{1}|\mathcal{H}(t, s)| d s$ for onward computation.

Define the operators

$$
\begin{aligned}
& \mathrm{F}: \mathcal{B} \rightarrow \mathcal{B}, \text { by }(\mathrm{Fu}) \mathrm{t}=\mathrm{l}(\mathrm{t}+\mathrm{m}) \mathrm{h}(\mathrm{u})+(1-\mathrm{l}(\mathrm{t}+\mathrm{m})) \mathrm{g}(\mathrm{u}), \\
& \mathrm{G}: \mathcal{B} \rightarrow \mathcal{B}, \text { by }(\mathrm{Gu}) \mathrm{t}=\int_{0}^{1} \mathcal{H}(\mathrm{t}, \mathrm{s}) \mathrm{f}(\mathrm{s}, \mathrm{u}(\mathrm{s})) \mathrm{ds}, \\
& \mathrm{T}: \mathcal{B} \rightarrow \mathcal{B}, \text { by }(\mathrm{Tu}) \mathrm{t}=(\mathrm{Fu}) \mathrm{t}+(\mathrm{Gu}) \mathrm{t} \text { or } \mathrm{u}(\mathrm{t})=(\mathrm{Tu}) \mathrm{t} .
\end{aligned}
$$

Thus the solutions of the considered BVP (1.1) are the fixed points of the operator equation (3.2).

To get our main results, we assume the following Lipschitz and growth conditions.

$\left(\mathrm{F}_{1}\right)$ For any $u, v \in \mathcal{B}$, with constants $\kappa_{h}, \kappa_{g} \in[0,1)$ which satisfy

$$
|g(u)-g(v)| \leqslant \kappa_{g}\|u-v\|_{c}, \quad \text { and } \quad|h(u)-h(v)| \leqslant \kappa_{h}\|u-v\|_{c} .
$$

$\left(F_{2}\right)$ For any $u \in \mathcal{B}$ there exist $C_{h}, C_{g}$ and $M_{g}, M_{h}>0$ such that

$$
|g(u)| \leqslant C_{g}\|u\|_{c}^{q_{1}}+M_{g}, \quad|h(u)| \leqslant C_{h}\|u\|_{c}^{q_{1}}+M_{h},
$$

where $\mathrm{q}_{1} \in[0,1)$.

$\left(F_{3}\right)$ For every $(t, u) \in J \times \mathbb{R}$, there exist $C_{f}, M_{f}>0, q_{2} \in[0,1)$, for which the following relation holds

$$
|f(t, u)| \leqslant C_{f}|u|^{q_{2}}+M_{f} .
$$

$\left(\mathrm{F}_{4}\right)$ For any $u, v \in \mathbb{R}$, we have some constant $\mathrm{L}_{\mathrm{f}}>0$ which yields

$$
|f(t, u(t))-f(t, v(t))| \leqslant L_{f}|u-v| .
$$

Theorem 3.2. The operator $\mathrm{F}$ is Lipschitz with $\Lambda=\max \left(\mathrm{\kappa}_{\mathrm{h}}, \mathrm{K}_{\mathrm{g}}\right)$ under the hypothesis $\left(\mathrm{F}_{1}\right)$. As a result, the operator $\mathrm{F}$ is $\alpha$-Lipschitz with constant $\Lambda$. Furthermore the growth condition $\|\mathrm{Fu}\|_{\mathrm{c}} \leqslant \mathrm{C}\|\mathrm{u}\|_{\mathrm{c}}^{\mathrm{q}_{1}}+\mathrm{M}$ holds for each $\mathrm{u} \in \mathcal{B}$, where $\mathrm{C}=\max \left(\mathrm{C}_{\mathrm{h}}, \mathrm{C}_{\mathrm{g}}\right)$ and $\mathrm{M}=\max \left(\mathrm{M}_{\mathrm{g}}, \mathrm{M}_{\mathrm{h}}\right)$. 
Proof. Taking, $u, v \in \mathcal{B}$ and considering

$$
|F u(t)-F v(t)| \leqslant l(m+1)|h(u)-h(v)|+(1-l(m+1))|g(u)-g(v)|,
$$

which implies that

$$
\begin{aligned}
\|F u-F v\|_{c} & \leqslant(m+1) l_{\kappa_{h}}\|u-v\|_{c}+(1-l(m+1)) \kappa_{g}\|u-v\|_{c} \\
& \leqslant \Lambda\|u-v\|_{c} .
\end{aligned}
$$

Showing that $F$ is Lipschitz with constant $\Lambda$ and also $\alpha$-Lipschitz with the same constant $\Lambda$. Further, for growth condition, we have

$$
\begin{aligned}
\|F u\|_{c} & \leqslant l(m+1)|h(u)|+(1-l(m+1))|g(u)| \\
& \leqslant l(m+1)\left(C_{h}\|u\|_{c}^{q_{1}}+M_{h}\right)+(1-l(m+1))\left(C_{g}\|u\|_{c}^{q_{1}}+M_{g}\right) \\
& \leqslant C\|u\|_{c}^{q_{1}}+M .
\end{aligned}
$$

By this we complete the proof.

Theorem 3.3. The operator $\mathrm{G}: \mathcal{B} \rightarrow \mathcal{B}$ is compact under the hypotheses $\left(\mathrm{F}_{2}\right),\left(\mathrm{F}_{3}\right)$, hence $\alpha$-Lipschitz with constant zero. Moreover $\mathrm{G}$ fulfills the growth condition given below

$$
\|G u\|_{\mathcal{c}} \leqslant \mathcal{H}^{*}\left(C_{\mathrm{f}}\|u\|_{\mathcal{C}}^{\mathbf{q}_{2}}+M_{\mathrm{f}}\right), \quad \mathrm{q}_{2} \in[0,1) .
$$

Proof. In order to prove that $\mathrm{G}$ is compact we first prove that $\mathrm{G}$ is continuous. To derive the continuity of $G$. Let us take a sequence $\left\{u_{n}\right\}$ in set $B_{k}=\left\{\|u\|_{c} \leqslant \mathcal{K}: u \in \mathcal{B}\right\} \subseteq \mathcal{B}$ with $u_{n} \rightarrow u$, as $n \rightarrow \infty$ in $B_{\mathcal{K}}$, where $B_{\mathcal{K}}$ is bounded. Then by continuity of $f\left(s, u_{n}(s)\right) \rightarrow f(s, u(s)), n \rightarrow \infty$ and by $\left(F_{3}\right)$, $\mathcal{H}(t, s)\left[f\left(s, u_{n}(s)\right)-f(s, u(s))\right] \leqslant \mathcal{H}(t, s) 2\left(C_{f} \mathcal{K}_{q_{2}}+M_{f}\right)$ and the function $s \rightarrow \mathcal{H}(t, s) 2\left(C_{f} \mathcal{K}^{q_{2}}+M_{f}\right)$ is integrable. From Lebesgue Dominated convergence theorem, one has

$$
\left|\left(G_{n} u\right) t-(G u) t\right| \leqslant \int_{0}^{1}|\mathcal{H}(t, s)|\left|f\left(s, u_{n}(s)\right)-f(s, u(s))\right| d s \rightarrow 0, \text { as } n \rightarrow \infty .
$$

To prove $G(D)$ is bounded for every $D \subseteq B_{\mathcal{K}}$, let $\left\{u_{n}\right\}$ be a sequence in $D$ then for every $u_{n} \in D$, we have

$$
\begin{aligned}
\left\|G u_{n}\right\|_{c} & \leqslant \int_{0}^{1}|\mathcal{H}(t, s)|\left|f\left(s, u_{n}(s)\right)\right| d s \\
& \leqslant \mathcal{H}^{*}\left(C_{f} \mathcal{K}^{q_{2}}+M_{f}\right) .
\end{aligned}
$$

which shows that $G(D)$ is bounded in $\mathcal{B}$. Next, we have to prove that $\left\{G u_{n}\right\}$ is equi-continuous. For $0 \leqslant t_{0}<t_{1} \leqslant 1$, we get

$$
\begin{aligned}
\left|\left(G u_{n}\right)\left(t_{0}\right)-\left(G u_{n}\right)\left(t_{1}\right)\right| & \left.=\int_{0}^{1} \mid \mathcal{H}\left(t_{0}, s\right)-\mathcal{H}\left(t_{1}, s\right)\right)|| f\left(s, u_{n}(s)\right) \mid d s \\
& \leqslant\left[C_{f}\left\|u_{n}\right\|^{q_{2}}+M_{f}\right] \int_{0}^{1}\left|\mathcal{H}\left(t_{0}, s\right)-\mathcal{H}\left(t_{1}, s\right)\right| d s \\
& \leqslant\left[C_{f}\left\|u_{n}\right\|^{q_{2}}+M_{f}\right]\left\{\left(t_{0}-t_{1}\right) \frac{l}{\Gamma(q)} \int_{0}^{1}(1-s)^{q-1} d s\right.
\end{aligned}
$$




$$
\begin{aligned}
& +\left(t_{1}-t_{0}\right) \frac{l}{\Gamma(q-p)} \int_{0}^{\eta}(\eta-s)^{q-p-1} d s+\left(t_{1}-t_{0}\right) \frac{l \lambda}{\Gamma(q-p)} \int_{0}^{\xi}(\xi-s)^{q-p-1} d s \\
& \left.+\frac{1}{\Gamma(q)}\left[\int_{0}^{t_{1}}\left(t_{1}-s\right)^{q-1} d s-\int_{0}^{t_{0}}\left(t_{0}-s\right)^{q-1} d s\right]\right\} \\
& \leqslant\left[C_{f} \mathcal{K}^{q_{2}}+M_{f}\right]\left\{\left(t_{0}-t_{1}\right) \frac{l}{\Gamma(q+1)}+\left(t_{1}-t_{0}\right) \frac{l}{\Gamma(q-p+1)} \eta^{q-p}\right. \\
& +\frac{\left(t_{1}-t_{0}\right) \lambda l}{\Gamma(q-p+1)} \xi^{q-p} \\
& \left.+\frac{1}{\Gamma(q)} \int_{0}^{t_{0}}\left(\left(t_{0}-s\right)^{q-1}-\left(t_{1}-s\right)^{q-1}\right) d s+\frac{1}{\Gamma(q)} \int_{t_{0}}^{t_{1}}\left(t_{1}-s\right)^{q-1} d s\right\}
\end{aligned}
$$

on simplification, we have

$$
\begin{aligned}
\left|\left(G u_{n}\right)\left(t_{0}\right)-\left(G u_{n}\right)\left(t_{1}\right)\right| \leqslant & \frac{\left[C_{f} \mathcal{K}^{q_{2}}+M_{f}\right]}{\Gamma(q+1)}\left\{l\left(t_{0}-t_{1}\right)+t_{0}^{q}-t_{1}^{q}+2\left(t_{1}-t_{0}\right)^{q}\right\} \\
& +\left[C_{f} \mathcal{K}^{q_{2}}+M_{f}\right]\left\{\left(t_{1}-t_{0}\right) \frac{l}{\Gamma(q-p+1)} \eta^{q-p}+\frac{\left(t_{1}-t_{0}\right) \lambda l}{\Gamma(q-p+1)} \xi^{q-p}\right\} .
\end{aligned}
$$

As $t_{0} \rightarrow t_{1}$, then right hand side of above equation (3.3) goes to 0. Therefore in light of Arzelä Ascolli Theorem, $\mathrm{G}$ is equi-continuous, hence $\mathrm{G}$ is completely continuous. Thus $\mathrm{G}$ is a compact operator. Further in view of Theorem 2.7, G is $\alpha$-Lipschitz with constant zero.

Theorem 3.4. Under the validity of assumptions $\left(\mathrm{F}_{1}\right)-\left(\mathrm{F}_{3}\right)$ and in view of Theorems 3.2 and 3.3, the operator $\mathrm{T}: \mathcal{B} \rightarrow \mathcal{B}$ is $\alpha$-Lipschitz with constant $\Lambda$. Hence $\mathrm{T}$ has at least one fixed point and the set of fixed points is bounded in $\mathcal{B}$.

Proof. Due to continuity of $F, G$, it is obvious that $T$ is also continuous. Since F, G are $\alpha$-Lipschitz with constant $\Lambda$ and 0 respectively. Therefore $T$ is also $\alpha$-Lipschitz with the same constant $\Lambda$. Further consider the set

$$
\mathbb{U}=\{u \in \mathcal{B}: \text { there exists } \lambda \in J \text { such that } u(t)=\lambda T u(t)\} .
$$

Now to prove that $\mathbb{U}$ is bounded subset of $\mathcal{B}$, we consider $u \in \mathbb{U}$ with $u=\lambda T u$, for $0 \leqslant \lambda \leqslant 1$, then

$$
\begin{aligned}
\|u\|_{c} & =\lambda\|T u\|_{c} \leqslant \lambda\left(\|F u\|_{c}+\|G u\|_{c}\right) \\
& \leqslant \lambda\left(C\|u\|_{c}^{q_{1}}+M+\mathcal{H}^{*}\left(C_{f}\|u\|_{\mathcal{c}}^{q_{2}}+M_{f}\right)\right),
\end{aligned}
$$

where $q_{1}, q_{2} \in[0,1)$, clearly $\mathbb{U}$ is bounded if not, let $\|u\|_{c} \rightarrow \infty$, then dividing the inequality (3.4) by $\|u\|_{c}$ and taking $\|\mathrm{u}\|_{\mathrm{c}} \rightarrow \infty$, we get

$$
1 \leqslant \lim _{\|u\| \rightarrow \infty} \frac{\lambda\left(C\|u\|_{\mathcal{c}}^{q_{1}}+M+\mathcal{H}^{*}\left(C_{f}\|u\|_{c}^{q_{2}}+M_{f}\right)\right)}{\|u\|_{c}}=0,
$$

which is contradiction. Consequently the operator $\mathrm{T}$ has at least one fixed point and the set of the fixed points of $\mathrm{T}$ is bounded in $\mathcal{B}$. Thus BVP (1.1) has at least one solution in $\mathbb{U}$ by using Theorem 2.9.

Remark 3.5. The following concluding remarks are obvious.

(i) If $\mathrm{q}_{1}=1$ the condition of Theorem 3.3 remains applicable provided $\mathrm{C}<1$.

(ii) If $\mathrm{q}_{2}=1$ the result of Theorem 3.3 holds provided that $\mathcal{H}^{*}\left(\mathrm{C}_{\mathrm{f}} \mathcal{K}+\mathrm{M}_{\mathrm{f}}\right)<1$. 
(iii) If $\mathrm{q}_{1}=\mathrm{q}_{2}=1$ then the conclusions of Theorem 3.3 remain applicable.

Theorem 3.6. Consider that the hypotheses $\left(\mathrm{F}_{1}\right)-\left(\mathrm{F}_{4}\right)$ hold. Then the BVP (1.1) has a unique solution with

$$
\Upsilon=2 m l \Lambda+(m+1) \frac{l L_{f}}{\Gamma(q+1)}+(1-m l) \frac{\delta L_{f}}{\Gamma(q-p+1)}+(m+1) \frac{L_{f} \lambda l}{\Gamma(q-p+1)}+\frac{L_{f}}{\Gamma(q+1)}<1 .
$$

Proof. Let $v(t)$ be another solution in $\mathcal{B}$, then

$$
\begin{aligned}
|T u(t)-T v(t)| \leqslant & l(m+1)|h u(t)-h v(t)|+(1-m l)|g u(t)-g v(t)| \\
& +(m+1) \frac{l}{\Gamma(q)}\left|\int_{0}^{1}(1-s)^{q-1}(f(s, u(s))-f(s, v(s))) d s\right| \\
& +(1-m l) \frac{\delta}{\Gamma(q-p)}\left|\int_{0}^{1}(1-s)^{q-p-1}[f(s, u(s))-f(s, v(s))] d s\right| \\
& +(m+1) \frac{\lambda l}{\Gamma(q-p)}\left|\int_{0}^{1}(1-s)^{q-p-1}[f(s, u(s))-f(s, v(s))] d s\right| \\
& +\frac{1}{\Gamma q}\left|\int_{0}^{t}(t-s)^{q-1}[f(s, u(s))-f(s, v(s))] d s\right|
\end{aligned}
$$

which implies that

$$
\begin{aligned}
\|T u-T v\| \leqslant & 2 m l \wedge\|u-v\|_{c} \\
& +\left[\frac{(m+1) L_{f}}{\Gamma(q+1)}+\frac{(1-m l) \delta L_{f}}{\Gamma(q-p+1)}+\frac{(m+1) L_{f} \lambda l}{\Gamma(q-p+1)}+\frac{L_{f}}{\Gamma(q+1)}\right]\|u-v\|_{c} \\
= & \Upsilon\|u-v\|_{c} .
\end{aligned}
$$

Hence, BVP (1.1) has a unique solution.

\section{Stability analysis of the solutions to boundary value problem (1.1)}

In this section, we study Hyers-Ulam and generalized Hyers-Ulam, Rassias stabilities for the solutions to the considered class of BVP (1.1) on the same fashion as studied in [9].

Theorem 4.1. Under the continuity of $\mathrm{f}$ and assumption $\left(\mathrm{F}_{4}\right)$ with $\mathrm{L}_{\mathrm{f}} \neq \frac{\Gamma(\mathrm{q}-\mathrm{p}+1)}{2}$, the solution of the class of BVP (1.1) is Hyers-Ulam stable and consequently, generalized Hyers-Ulam stable.

Proof. Let $u \in \mathcal{B}$ be a solution of (1.1) and $v \in \mathcal{B}$ be the unique solution of (1.1)

$$
\left\{\begin{array}{l}
{ }^{c} D^{q} u(t)=f(t, u(t)), \quad 1<q \leqslant 2, \quad t \in J=[0,1], \\
u(0)=v(0), \quad u(1)=v(1),
\end{array}\right.
$$

where $0<p<1, f: J \times \mathbb{R} \rightarrow \mathbb{R}$. The general solution is given by

$$
u(t)=l(t+m) h(v)+(1-l(t+m)) g(v)+\int_{0}^{1} \mathcal{H}(t, s) f(s, v(s)) d s
$$

From which, we have

$$
\left|u(t)-\left(l(t+m) h(u)+(1-l(t+m)) g(u)+\int_{0}^{1} \mathcal{H}(t, s) f(s, u(s)) d s\right)\right| \leqslant \varepsilon .
$$


Hence in view of the aforesaid relation, we have

$$
\begin{aligned}
|u(t)-v(t)|= & \left|u(t)-\left(l(t+m) h(v)+(1-l(t+m)) g(v)+\int_{0}^{1} \mathcal{H}(t, s) f(s, v(s)) d s\right)\right| \\
\leqslant & \left|u(t)-\left(l(t+m) h(u)+(1-l(t+m)) g(u)+\int_{0}^{1} \mathcal{H}(t, s) f(s, u(s)) d s\right)\right| \\
& +\left|\int_{0}^{1} \mathcal{H}(t, s) f(s, u(s)) d s-\int_{0}^{1} \mathcal{H}(t, s) f(s, v(s)) d s\right| \\
\leqslant & \varepsilon+\frac{2 L_{f}|u(t)-v(t)|}{\Gamma(q-p+1)} \\
|u(t)-v(t)| \leqslant & \frac{\varepsilon}{1-\frac{2 L_{f}}{\Gamma(q-p+1)}}=C_{f} \mathcal{E},
\end{aligned}
$$

where $\mathrm{L}_{\mathrm{f}} \neq \frac{\Gamma(\mathrm{q}-\mathrm{p}+1)}{2}, \mathrm{C}_{\mathrm{f}}=\frac{1}{1-\frac{2 \mathrm{~L}_{\mathrm{f}}}{\Gamma(\mathrm{q}-\mathrm{p}+1)}}$.

Therefore solution of the BVP (1.1) is Hyers-Ulam stable. Further by using $\Phi_{f}(\varepsilon)=C_{f} \varepsilon, \Phi_{f}(0)=0$ implies that solution of (1.1) is generalized Hyers-Ulam stable.

Theorem 4.2. Assume that $\mathrm{f}$ is continuous and assumption $\left(\mathrm{F}_{4}\right)$ satisfies with $\mathrm{L}_{\mathrm{f}} \neq \frac{\Gamma(\mathrm{q}-\mathrm{p}+1)}{2}$. If there exists $\phi(t) \in \mathrm{C}\left(\mathrm{J}, \mathbb{R}^{+}\right)$satisfies (2.2), then the solution of BVP (1.1) is Hyers-Ulam Rassias stable and consequently generalized Hyers-Ulam-Rassias stable.

Proof. Let $u \in \mathcal{B}$ be any solution of (1.1), then

$$
\left|u(t)-\left(l(t+m) h(u)+(1-l(t+m)) g(u)+\int_{0}^{1} \mathcal{H}(t, s) f(s, u(s)) d s\right)\right| \leqslant \varepsilon \phi(t) .
$$

Therefore in view of relation (4.1), for solution $v \in \mathcal{B}$, we impose

$$
\begin{aligned}
|u(t)-v(t)|= & \left|u(t)-\left(l(t+m) h(v)+(1-l(t+m)) g(v)+\int_{0}^{1} \mathcal{H}(t, s) f(s, v(s)) d s\right)\right| \\
\leqslant & \left|u(t)-\left(l(t+m) h(u)+(1-l(t+m)) g(u)+\int_{0}^{1} \mathcal{H}(t, s) f(s, u(s)) d s\right)\right| \\
& +\left|\int_{0}^{1} \mathcal{H}(t, s) f(s, u(s)) d s-\int_{0}^{1} \mathcal{H}(t, s) f(s, v(s)) d s\right| \\
\leqslant & \varepsilon \phi(t)+\frac{2 L_{f}|u(t)-v(t)|}{\Gamma(q-p+1)}, \\
|u(t)-v(t)| \leqslant & \frac{\varepsilon \phi(t)}{1-\frac{2 L_{f}}{\Gamma(q-p+1)}}=\phi(t) \varepsilon, \quad L_{f} \neq \frac{\Gamma(q-p+1)}{2} .
\end{aligned}
$$

So in view of the above result, solution of BVP (1.1) is Hyers-Ulam-Rassias stable. Further on the same fashion, it can be shown that the solution of BVP (1.1) is generalized Hyers-Ulam-Rassias stable.

\section{Example}

\section{Example 5.1.}

$$
\left\{\begin{array}{l}
{ }^{c} D^{\frac{3}{2}} u(t)=\frac{\sin ^{2} t|u(t)|^{\frac{1}{2}}}{\left(9+e^{2 t}\right)\left(1+|u(t)|^{\frac{1}{2}}\right)}, \quad t \in[0,1] \\
u(0)=\frac{1}{2} D^{\frac{1}{2}} u\left(\frac{3}{10}\right)+\sum_{i=1}^{5} \lambda_{i}\left|u\left(t_{i}\right)\right| \\
u(1)=\frac{1}{5} D^{\frac{1}{2}} u\left(\frac{1}{5}\right)+\sum_{i=1}^{10} \lambda_{i}\left|u\left(t_{i}\right)\right|, \quad \sum_{i=1}^{10} \lambda_{i}<1, \quad \lambda_{i}>0,
\end{array}\right.
$$


Define

$$
\begin{aligned}
f(t, u) & =\frac{\sin ^{2} t|u(t)|^{\frac{1}{2}}}{\left(9+e^{2 t}\right)\left(1+|u(t)|^{\frac{1}{2}}\right)}, \quad(t, u) \in[0,1] \times[0, \infty), \\
g(u) & =\sum_{i=1}^{5} \lambda_{i}\left|u\left(t_{i}\right)\right|, \quad h(u)=\sum_{i=1}^{10} \lambda_{i}\left|u\left(t_{i}\right)\right| .
\end{aligned}
$$

Then

$$
\begin{aligned}
|f(t, u)| & \leqslant \frac{1}{10} \frac{|u(t)|^{\frac{1}{2}}}{1+|u(t)|^{\frac{1}{2}}} \leqslant \frac{1}{10}|u(t)|^{\frac{1}{2}} \\
& \leqslant C_{f}|u|^{q_{2}}+M_{f},
\end{aligned}
$$

with $C_{f}=\frac{1}{10}, q_{2}=\frac{1}{2}, M_{f}=0$, and

$$
|g(u)|=\sum_{i=1}^{5} \lambda_{i}\left|u\left(t_{i}\right)\right| \leqslant C_{g}\|u\|^{q_{1}}+M_{g}
$$

with $\mathrm{C}_{\mathrm{g}}=\sum_{i=1}^{5} \lambda_{\mathrm{i}}<1, \mathrm{q}_{1}=1, \mathrm{M}_{\mathrm{g}}=0$.

Also $|h(u)| \leqslant C_{h}\|u\|^{q_{1}}+M_{h}$ with $C_{h}=\sum_{i=1}^{10} \lambda_{i}<1, M_{h}=0$. Further

$$
|g(u)-g(v)| \leqslant \sum_{i=1}^{5} \lambda_{i}\|u-v\| \leqslant \kappa_{g}\|u-v\|_{c}, \quad \kappa_{g}=\sum_{i=1}^{5} \lambda_{i}<1,
$$

and

$$
|h(u)-h(v)| \leqslant \sum_{i=1}^{10} \lambda_{i}\|u-v\|_{c} \leqslant \kappa_{h}\|u-v\|_{c}, \quad \kappa_{h}=\sum_{i=1}^{5} \lambda_{i}<1 .
$$

Here $\mathrm{q}_{1}=1$ and $\mathrm{C}=\max \left(\mathrm{C}_{\mathrm{g}}, \mathrm{C}_{\mathrm{h}}\right)=\sum_{i=1}^{10} \lambda_{i}<1$.

In view of these quantities and using $q=\frac{3}{2}, p=\frac{1}{2}, L_{f}=\frac{1}{10}, \delta=\frac{1}{2}, \lambda=\frac{1}{5}$, it is easy to prove that BVP (5.1) has a unique solution by using Theorem 3.6. It is also obvious that the solution of BVP (5.1) is Hyers-Ulam stable, generalized Hyers-Ulam stable and Hyers-Ulam-Rassias stable by using Theorem 4.1 and 4.2 respectively.

\section{Acknowledgment}

This project was supported by the Theoretical and Computational Science (TaCS) Center under Computational and Applied Science for Smart Innovation Cluster (CLASSIC), Faculty of Science, KMUTT. We are really thankful to reviewers for their useful comments which improved the quality of the paper.

This research paper has been financially supported by the institute for Mathematical Research, University Putra Malaysia, 43400 UPM Serdang, Selangor, Malaysia.

\section{References}

[1] M. I. Abbas, Existence and uniqueness of solution for a boundary value problem of fractional order involving two Caputo's fractional derivatives, Adv. Difference Equ., 2015 (2015), 12 pages. 1 
[2] B. Ahmad, J. J. Nieto, Existence results for a coupled system of nonlinear fractional differential equations with three-point boundary conditions, Comput. Math. Appl., 58 (2009), 1838-1843. 1

[3] A. Ali, B. Samet, K. Shah, R. A. Khan, Existence and stability of solution to a toppled systems of differential equations of non-integer order, Bound. Value Probl., 2017 (2017), 13 pages. 1

[4] M. Benchohra, J. E. Lazreg, On stability for nonlinear implicit fractional differential equations, Matematiche (Catania), 70 (2015), 49-61. 1

[5] K. Deimling, Nonlinear functional analysis, Springer-Verlag, Berlin, (1985). 2, 2

[6] F. B. M. Duarte, J. A. Tenreiro Machado, Chaotic phenomena and fractional-order dynamics in the trajectory control of redundant manipulators. Fractional order calculus and its applications, Nonlinear Dynam., 29 (2002), 315-342. 1

[7] Z.-Y. Gao, X.-L. Yu, J.-R. Wang, Exp-type Ulam-Hyers stability of fractional differential equations with positive constant coefficient, Adv. Difference Equ., 2015 (2015), 14 pages. 1

[8] D. H. Hyers, On the stability of the linear functional equation, Proc. Nat. Acad. Sci. U. S. A., 27 (1941), 222-224. 1

[9] R. W. Ibrahim, Ulam stability of boundary value problem, Kragujevac J. Math., 37 (2013), 287-297. 4

[10] F. Isaia, On a nonlinear integral equation without compactness, Acta Math. Univ. Comenian. (N.S.), 75 (2006), $233-240$. $1,2,2$

[11] S.-M. Jung, Hyers-Ulam stability of linear differential equations of first order, II, Appl. Math. Lett., 19 (2006), 854-858. 1

[12] R. A. Khan, K. Shah, Existence and uniqueness of solutions to fractional order multi-point boundary value problems, Commun. Appl. Anal., 19 (2015), 515-526. 1

[13] A. A. Kilbas, H. M. Srivastava, J. J. Trujillo, Theory and applications of fractional differential equations, North-Holland Mathematics Studies, Elsevier Science B.V., Amsterdam, (2006). 1, 2

[14] H.-L. Li, Y.-L. Jiang, Z.-L. Wang, L. Zhang, Z.-D. Teng, Global Mittag-Leffler stability of coupled system of fractionalorder differential equations on network, Appl. Math. Comput., 270 (2015), 269-277. 1

[15] J. Mawhin, Topological degree methods in nonlinear boundary value problems, Expository lectures from the CBMS Regional Conference held at Harvey Mudd College, Claremont, Calif., June 9-15, 1977, CBMS Regional Conference Series in Mathematics, American Mathematical Society, Providence, R.I., (1979). 1

[16] K. S. Miller, B. Ross, An introduction to the fractional calculus and fractional differential equations, A Wiley-Interscience Publication, John Wiley \& Sons, Inc., New York, (1993). 1, 2

[17] M. Obloza, Hyers stability of the linear differential equation, Rocznik Nauk.-Dydakt. Prace Mat., 13 (1993), 259-270. 1

[18] I. Podlubny, Fractional differential equations, An introduction to fractional derivatives, fractional differential equations, to methods of their solution and some of their applications, Mathematics in Science and Engineering, Academic Press, Inc., San Diego, CA, (1999). 1, 2

[19] T. M. Rassias, On the stability of the linear mapping in Banach spaces, Proc. Amer. Math. Soc., 72 (1978), 297-300. 1

[20] B. Ricceri, On the Cauchy problem for the differential equation $f\left(t, x, x^{\prime}, \ldots, x^{(k)}\right)=0$, Glasgow Math. J., 33 (1991), 343-348. 1

[21] I. A. Rus, Ulam stabilities of ordinary differential equations in a Banach space, Carpathian J. Math., 26 (2010), $103-107$. 1

[22] K. Shah, R. A. Khan, Multiple positive solutions to a coupled systems of nonlinear fractional differential equations, SpringerPlus, 5 (2016), 20 pages. 1

[23] K. Shah, S. Zeb, R. A. Khan, Existence and uniqueness of solutions for fractional order m-point boundary value problems, Fract. Differ. Calc., 5 (2015), 171-182. 1

[24] S. Phiangsungnoena, W. Sintunavarat, P. Kumam, Fixed point results for generalized Ulam-Hyers stability and wellposedness via $\alpha$-admissible in b-metric spaces, Fixed Point Theory and Appl., 2014 (2014), 17 pages. 1

[25] S. Phiangsungnoen, P. Kumam, Generalized Ulam-Hyers stability and well posedness for fixed point equation via $\alpha$ admissible, J. Inequal. Appl., 2014 (2014), 12 pages. 1

[26] S. M. Ulam, Problems in modern mathematics, Science Editions John Wiley \& Sons, Inc., New York, (1964). 1

[27] J.-R. Wang, L.-L. Lv, Y. Zhou, Ulam stability and data dependence for fractional differential equations with Caputo derivative, Electron. J. Qual. Theory Differ. Equ., 2011 (2011), 10 pages. 1

[28] J.-R. Wang, Y.-R. Zhang, Existence and stability of solutions to nonlinear impulsive differential equations in $\beta$-normed spaces, Electron. J. Differential Equations, 2014 (2014), 10 pages. 1

[29] J.-R. Wang, Y. Zhou, W. Wei, Study in fractional differential equations by means of topological degree methods, Numer. Funct. Anal. Optim., 33 (2012), 216-238. 1

[30] J. R. L. Webb, S. C. Welsh, Existence and uniqueness of initial value problems for a class of second-order differential equations, J. Differential Equations, 82 (1989), 314-321. 1

[31] K. Wongkum, P. Chaipunya, P. Kumam, On the generalized Ulam-Hyers-Rassias stability of quadratic nappings in modular spaces without $\Delta_{2}$-conditions, J. Funct. Spaces, 2015 (2015), 6 pages. 1 